\title{
A Comparative Study of Infomax, Extended Infomax and Multi-User Kurtosis Algorithms for Blind Source Separation
}

\author{
Monorama Swaim, Silicon Institute of Technology, Bhubaneswar, IN \\ Rutuparna Panda, Veer Surendra Sai University of Technology, Odisha, IN \\ Prithviraj Kabisatpathy, C. V. Raman College of Engineering, Odisha, IN
}

\begin{abstract}
In this article for the separation of Super Gaussian and Sub-Gaussian signals, we have considered the Multi-User Kurtosis(MUK), Infomax (Information Maximization) and Extended Infomax algorithms. For Extended Infomax we have taken two different non-linear functions and new coefficients and for Infomax we have taken a single non-linear function. We have derived MUK algorithm with stochastic gradient update iteratively using MUK cost function abided by a Gram-Schmidt orthogonalization to project on to the criterion constraint. Amongst the various standards available for measuring blind source separation, Cross-correlation coefficient and Kurtosis are considered to analyze the performance of the algorithms. An important finding of this study, as is evident from the performance table, is that the Kurtosis and Correlation coefficient values are the most favorable for the Extended Infomax algorithm, when compared with the others.
\end{abstract}

\section{KEYWORDS}

Blind Source Separation, Correlation Coefficient, Extended Infomax, Information Maximization (Infomax), Kurtosis, Multi-User Kurtosis

\section{INTRODUCTION}

Over the past few decades, the Blind Source Separation (BSS) problem has found wide attention in the area of speech and signal processing, communication and neuroscience. The Blind source separation problem recovers the original sources from their mixtures without any prior information about the input sources (Amari, 1998). In BSS there are different approaches are considered, one approach is instantaneous BSS and the other one is convolutive BSS. In both cases the nature of mixing process are different. Here we have considered the number of outputs similar to the number of input sources and the mixing process is instantaneous. Different researchers have studied the BSS problem in different areas such as neural network and statistical signal processing. It is necessary for a blind source separation algorithm to separate the signal sources of different distributions, but due to some significantly fluctuating parameter values most of the algorithms failed to separate the sources. The Information maximization approach which was developed by Bell and T. Sejnowski based on entropy maximization approach can separate the signals those have super Gaussian distributions and fails to 
separate the negative kurtosis values of a signal. It motivated the researchers to develop the extended Information maximization approach which is able to separate the signals those have super-Gaussian and sub Gaussian distributions (Lee, Girolami, \& Sejnowski, 1999). Here the performance of the source separation is evaluated by different quality measures: such as k, co-relation coefficient and SIR which are one of the essential parameter measurement of quality of separation. Researchers Soloumiac and Cardoso (1993), Comon (1994) and Garrat and Pham (1997) proposed some of the algorithms which also separate mixtures of Supergaussian and Sub Gaussian sources. Also, C. B Papadias (2000) suggested another algorithm based on higher order statistics i.e multiuser kurtosis algorithm; this can separate the signals of Gaussian and super-Gaussian signals. Here we have compared Information maximization algorithm, Extended Infomax and Multi-User Kurtosis maximization algorithms, based on source separation. Also, we have obtained the results for blind deconvolution of one speech signal, for both Infomax and Extended Infomax algorithms.

The paper is orchestrated as follows; Section 2 describes problem formulation. In section 3, the information maximization approach is discussed. In sections 4 and 5, we describe the different algorithms used for blind source separation. In section 6 all the experimental procedures and the corresponding results are described in detail. Section 7 concludes the study.

\section{METHODOLOGY}

We have passed signal $S$ to the model which is statistically independent, then the signal mixed with matrix A to get result vector Y. After that matrix W is calculated using de-mixing (Routray \& Kishore, 2007).

The BSS model as in equation (1) below

$$
X(r)=A S(r)+v(r)
$$

Where

$S(r)=\left[s_{1}(r), s_{2}(r), \ldots, s_{p}(r)\right]^{T}$ transmitted Source Signals vector

$A:$ is Mixing Matrix.

$X(r):$ is a vector (acquired signal)

$v(r)$ : Vector with add-on noise samples at time instant $r$

$T$ is Matrix / Transpose Vector

Figure 1 shows schematic overview of BSS model. We have considered six audio sources of different distributions, primarily sub-Gaussian and super-Gaussian using Multiuser kurtosis, Information maximization and Extended Information maximization algorithms (Comon \& Rota, 2003) for the BSS. Here we have considered the number of source signals equal to the number of sensors. In earlier studies there were various algorithms already developed for BSS. In our model, we have taken the extended Infomax, one of the successful neural network based approaches in separating super-Gaussian signals, in addition to Infomax and multiuser kurtosis algorithm. Subsequently the cross-correlation coefficient and kurtosis were computed for performance measurement in-between the input data and output data.

The six sources $s_{1}(r), s_{2}(r), s_{3}(r), s_{4}(r), s_{5}(r), s_{6}(r)$ are mixed through a mixing matrix $A$, gives observed mixed signals $X_{1}(r), X_{2}(r), X_{3}(r), X_{4}(r), X_{5}(r), X_{6}(r)$ (Lee, Girolami, \& 


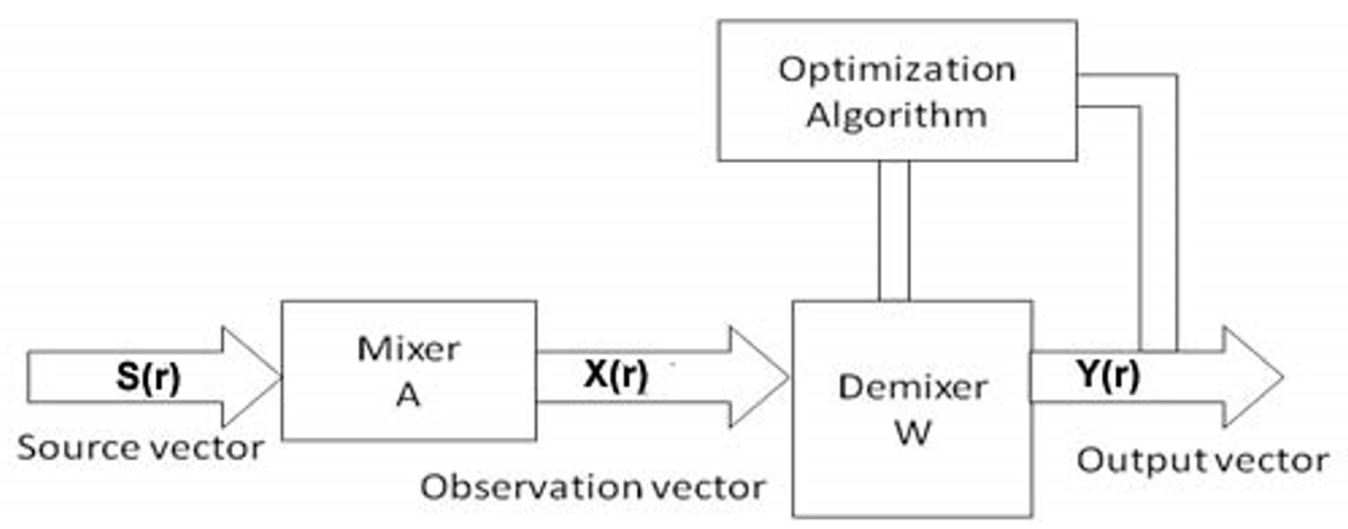

Sejnowski, 1999; Papadias, 2000). The objective of source separation algorithm to get all source signals (Kadou \& Arakawa, 2009). To find the demixing matrix $W^{T}$ from source signal $S(r)$ Equation (2) shows the components of the reconstructed signals.

The plan is to get the de-mixing matrix $W^{T}$ having the components of the reconstructed signals are mutually independent without the knowledge of $A$ and distribution of the source signal $S(r)$, is given in the below equation

$Y(r)=W^{T}(r) X(r)$

\section{INFORMATION MAXIMIZATION APPROACH}

A. J. Bell and T. J. Sejnowski, (1995-1997) had formulated the Infomax, since then it is treated one of the self-organizing learning methods for getting the maximum mutual information. The output $Y$ of Neural Network processor for the input $\mathrm{X}$ defined as below

$$
I(Y, X)=H(Y)-H(Y \mid X)
$$

Where $H(Y)$ is the entropy of the output and $H(Y \mid X)$ is the entropy of the output not consider within the input.

The scaling between $\mathrm{X}$ and $\mathrm{Y}$ is deterministic and $H(Y \mid X)$ is lowest possible measure which deviates to $-\infty$. The equation (3) can be defined as below, based on the parameter $w$, the calibration can be from $X$ to $Y$.

$$
\frac{\partial}{\partial w} I(Y, X)=\frac{\partial}{\partial w} H(Y)
$$

As does not depends on, its derivative is zero. The entropy of output can be derived as 
$(Y)=-E\left[\ln f_{y}(y)\right]=-\int_{-x}^{x} f_{y}(y) \ln f_{y}(y) d y$

$E[$.$] gives excluded value and f_{y}(y)$ is the probability density function of the output. For having multiple input and output system, A. J. Bell and T. J. Sejnowski considered an input $x$ weight matrix $W$, bias vector $w_{0}$ and a monotonically transformed output vector

$y=g\left(W_{x}+w_{0}\right)$

The probability function of $y$ can be defined as

$f_{y}(y)=\frac{f_{x}(x)}{|J|}$

Where $|J|$ is Jacobian transformation.

In-order to maximize entropy we have to maximize $\ln |J|$.

To consider logistic function as the non-linearity, i.e. $g(u)=\left(1+e^{u}\right)^{-1}, u=W_{x}+w_{0}$ the resulted learning rules will be

$$
\begin{aligned}
& \frac{d H(y)}{d W}=W^{-T}+(1-2 y) x^{T} \\
& \frac{d H(y)}{d w_{0}}=1-2 y
\end{aligned}
$$

\section{MULTI USER KURTOSIS (MUK) MAXIMIZATION ALGORITHM}

In this work we have considered a MUK algorithm for solving the BSS problem. From the BSS model, we know that $Y(r)=W^{T}(r) X(r)$

Which can be formulated as?

$$
Y(r)=W^{T}(r) A S(r)=G^{T}(r) S(r)
$$

Where $W(r)$ is $n \times m$ matrix and $G^{T}(r) m \times m$ global response matrix.

Here we have implemented the algorithm for the separation of super-Gaussian and subgaussian signals (Papadias, 2000). 
If each $s_{j}(r),(\mathrm{j}=1,2, \ldots, \mathrm{p})$ is non Gaussian and zero-mean sequence $\left\{s_{j}(r)\right\},\left\{s_{g}(r)\right\}$ are statistically independent for $j \neq g$, the conditions considered for recovery of source or transmitted signals at the outputs:

1. $\left|K Y_{j}(r)\right|=\left|K_{s}\right|, j=1,2 \ldots \ldots p$

2. $\mathrm{K}\left|Y_{j}(r)\right|^{2}=\sigma_{s}^{2}, j=1,2 \ldots . p$

3. $E\left(Y_{j}(r) Y_{r}^{*}(r)\right)=0$, where $j \neq 1$

Where $E(x)=E\left(|x|^{4}\right)-2 E^{2}\left(|x|^{2}\right)-\left|E\left(x^{2}\right)\right|$ is the (un-normalized) kurtosis of $x, \sigma_{s}{ }^{2}, K_{s}$ are the variance and kurtosis of each $s_{j}(r)$ respectively and $*$ marks complex conjugate.

The BSS prototype, which is, derived from conditions (I), (2) and (3).

$$
\left\{\begin{array}{l}
{ }_{\mathrm{G}}^{\max } \mathrm{F}(\mathrm{G})=\sum_{\mathrm{j}=1}^{\mathrm{p}}\left|\mathrm{K}\left(\mathrm{Y}_{\mathrm{j}}\right)\right| \\
\text { subjectto } \mathrm{G}^{\mathrm{H}} G=\mathrm{I}_{\mathrm{p}}
\end{array}\right.
$$

Where $I_{p}$, is the $p \times p$ identity matrix and $\mathrm{H}$ marks Hermitian transpose. The restriction comes from the manifest that, according to (2) and (3), $\mathrm{E}\left(\mathrm{YY}^{\mathrm{H}}\right)=\tilde{\mathrm{A}}_{\mathrm{s}}^{2} \mathrm{I}_{\mathrm{p}}$ (computed that $\tilde{\mathrm{A}}_{\mathrm{s}}^{2}=1$ ). We have taken this equation as Multi-User Kurtosis (MUK) maximization basis for blind source separation. The steps carried out for implementation of MUK algorithm as:

1. Update $W(r)$, instantaneous gradient (by a step size $\mu$ ):

$$
W^{\prime}(r+1)=W(r)+\mu \operatorname{sign}\left(K_{s}\right) X^{*}(r) Y(r)
$$

2. Continuing the iteration to satisfy the orthogonality basis:

$G^{H}(r+1) G(r+1)=I_{p}$. Considering $H$ is unitary it gives

$W^{H}(r+1) W(r+1)=I_{p}$

3. For $\mathrm{j}=1, \ldots, \mathrm{p}(14)$

Where $\delta_{r j}$ is kronecker delta, Equation (13) can be written as

$\left\{\begin{array}{l}\min _{W_{j}} \Delta\left(W_{j}\right)=\left(W_{j}-W^{\prime}{ }_{j}\right)^{H}\left(W_{j}-W^{\prime}{ }_{j}\right) \\ \text { subject to: } W_{l}^{H} W_{j}=\delta, l=1, \ldots, j\end{array}\right.$ 
4. In order to derive the Equation (15) the Lagrangian of $\Delta\left(W_{j}\right)$ as follow

$$
L_{\Delta\left(w_{j}, \lambda_{j}, \mu_{j}, v_{j}\right)}=\Delta W_{j}-\lambda_{j}\left(W_{j}^{H} W_{j}-1\right)-\sum_{r=1}^{j-1} \mu_{r j} \operatorname{Re}\left(W_{r}^{H} W_{j}\right)-\sum_{r}^{j-1} v_{r j} \operatorname{Im}\left(W_{r}^{H} W_{j}\right)
$$

MUK algorithm:

1. $\mathrm{r}=0$ : initialize $\mathrm{W}(0)=\mathrm{W}_{0}$

2. for $r>0$

3. Obtain $\mathrm{W}^{\prime}(\mathrm{r}+1)$ from (12)

4. Obtain $\mathrm{W}_{1}(\mathrm{r}+1)=\mathrm{W}_{1}^{\prime}(\mathrm{r}+1) / \mathrm{W}_{1}^{\prime}(\mathrm{r}+1)$

5. for $\mathrm{j}=2: \mathrm{p}$

6. Calculate $\mathrm{W}_{\mathrm{j}}(\mathrm{r}+1)$ from (21)

7. Go to 5

8. $\mathrm{W}(\mathrm{r}+1)=\left[\mathrm{W}_{1}(\mathrm{r}+1) \ldots \mathrm{W}_{\mathrm{p}}(\mathrm{r}+1)\right]$

9. Go to 2

5. Where $\lambda_{j}, \mu_{r j}, v_{r j}$ real scalars. the gradient of $L_{\Delta\left(w_{j}, \lambda_{j}, \mu, v\right)}$ with respect to $W_{j}$ to zero $\left(\frac{d L_{\Delta}}{d W_{j}^{\prime}}=0\right)$, so for each $\mathrm{j}$ the equation will be:

$W_{j}-W_{j}^{\prime}-\lambda_{j} W_{j}-\int_{r=1}^{j-1} \beta_{r j} W_{r}=0$

Where $\beta_{r j}=1 / 2\left(\mu_{r j}+i v_{r j}\right)$ and $i=\sqrt{-1}$ from (17)

$\left\{\begin{array}{l}\lambda_{j}=1-W_{j}^{W} W_{j}^{\prime} \\ \beta_{l j}=-W_{l}^{H} W_{j}^{\prime}\end{array}\right.$

Which gives $\left(1-\lambda_{j}\right) W_{j}=W_{j}^{\prime}-\sum_{r=1}^{j-1} W_{r}^{H} W_{j}^{\prime} W_{r}$

According to (19),

$W_{j} \propto\left(W_{j}^{\prime}-\sum_{r=1}^{j-1} W_{r}^{H} W_{j}^{\prime} W_{r}\right)$ 
Where $\propto$ denotes "proportional."

Now

$$
W_{j}(r+1)=\frac{W_{j}^{\prime}(r+1)-\sum_{r=1}^{j-1}\left(W_{r}^{H}(r+1) W_{j}^{\prime}(r+1)\right) W_{r}(r+1)}{W_{j}^{\prime}(r+1)-\sum_{r=1}^{j-1}\left(W_{r}^{H}(r+1) W_{j}^{\prime}(r+1)\right) W_{r}(r+1)}
$$

The matrix $W_{j}^{\prime}(r+1)$ (Gram-Schmidt Orthogonalization), carried out in steps 4 to 8 in the above algorithm.

\section{EXTENDED INFORMATION MAXIMIZATION APPROACH}

The extended Infomax technique is the algorithm which is the extended version of Infomax. According to various sources (Lee, Girolami, \& Sejnowski, 1999; Minhas \& Gaydecki, 2014) it has been observed that the extended Infomax can separate ample number of sources arising from a variety of distributions. In Infomax only one linear function is used in the learning rule but in Extended infomax Bell and T. Sejnowski, 1995-97) have considered two learning rules for super Gaussian and sub-Gaussian signals. Here we have utilized both the rules for our study.

$\Delta W \propto\left[1-\operatorname{Ktanh}(u) u^{T}-u u^{T}\right] W$

$\Delta W \propto\left[1+K \tanh (u) u^{T}-u u^{T}\right] W$

Here K represents the kurtosis values like 1 and -1 for super-Gaussian and sub-Gaussian signals.

\section{EXPERIMENTAL SET UP AND ANALYSIS OF RESULTS}

\subsection{Blind Source Separation Results}

The simulations and experiments were executed on recorded dataset to verify the performance of the three algorithms i.e. Infomax, Extended Infomax and Multi-user Kurtosis. We have observed that the algorithms can separate the source (signals) with variety of distributions (sub-gaussian, Super Gaussian) as shown in Figures 2, 3, and 4; however, there are very small variations in the performance, as shown by Table 1 , which contain kurtosis values and Tables 2, 3, and 4 shows cross co-relation coefficient values of three algorithms. We have taken 6 sources of different distributions which were successfully separated. The learning rate values of 0.001 and 0.005 have been chosen for the experiment. If the coefficient is nearer to either 1 or -1 , then the correlation between the variables is resulted to be stronger. We compared the performance of the Infomax, Multi user kurtosis and Extended infomax for the source separation (Comon, 1994; Bell \& Sejnowski, 1995; Diamantaras, Vranou, \& Papadimitriou, 2013; Swain, Biswal, Panda, \& Kabisatpathy, 2017).

\subsection{Blind Deconvolution Results}

We have choosen a single unknown speech signal for Infomax and Extended infomax

In the Blind Deconvolution procedure for Infomax and Extended infomax we have selected a single unknown speech signal $\mathrm{S}(r)$ which is convolved with an unknown tapped delay line filter 
Table 1. Kurtosis of six original sources and estimated signal sources from Infomax, Extended Infomax, and Multi-user kurtosis

\begin{tabular}{|c|c|c|c|c|c|}
\hline Source number & Source type & $\begin{array}{c}\text { Original } \\
\text { kurtosis }\end{array}$ & $\begin{array}{c}\text { Estimated } \\
\text { kurtosis(Infomax) }\end{array}$ & $\begin{array}{c}\text { Estimated kurtosis } \\
\text { (Extended Infomax) }\end{array}$ & $\begin{array}{c}\text { Estimated } \\
\text { kurtosis (Multi- } \\
\text { user kurtosis) }\end{array}$ \\
\hline 1 & Speech 1 & 8.633 & 8.5252 & 8.6973 & 8.6732 \\
\hline 2 & Music 1 & 2.4336 & 2.3347 & 2.4786 & 2.3333 \\
\hline 3 & Speech 3 & 10.348 & 9.435 & 10.3535 & 10.3312 \\
\hline 4 & Speech 4 & 11.678 & 10.667 & 11.6432 & 10.8569 \\
\hline 5 & Speech 5 & 12.834 & 11.8675 & 12.8355 & 12.4456 \\
\hline 6 & Sound1 & 1.2651 & 1.0975 & 1.2555 & 1.1775 \\
\hline
\end{tabular}

Table 2. Cross co-relation coefficient for Infomax

\begin{tabular}{|l|l|}
\hline 1.0000 & 0.9963 \\
\hline 0.9998 & 0.9989 \\
\hline
\end{tabular}

Table 3. Cross co-relation coefficient for Extended Infomax

\begin{tabular}{|l|l|}
\hline 1.0000 & 1.0000 \\
\hline 1.0000 & 1.0000 \\
\hline
\end{tabular}

Table 4. Cross co-relation coefficient for Multiuser Kurtosis

\begin{tabular}{|l|l|}
\hline 0.9999 & 1.0000 \\
\hline 1.0000 & 0.9998 \\
\hline
\end{tabular}

$c_{1}, c_{2}, \ldots \ldots, c_{N}$ resulting in a corrupt signal $x(r)=a(r) * s(r)$ i.e. $c(r)$ is the impulse response of the filter. The task is to recover $\mathrm{S}(r)$ by convolving $X(r)$ with a known filter $w_{1}, w_{2}, \ldots \ldots, w_{m}$ which switches the effect of the filter $a(r)$. Speech signals were convolved with various filters and learning rules were used to execute blind deconvolution (Bell \& Sejnowski, 1995, 1997). Here we have chosen the learning rate 0.0001 for better evaluation. From the experiment for both Infomax and Extended infomax we have displayed the convolving filter, deconvolving filter and learnt filter, and the spectrogram for Infomax and extended Infomax as shown in Figures 5-8. Here a 6.25ms echo added to the signal, that sounds a mild audible barrel effect. From the plots it has been shown that the resulting deconvolution of extended infomax shown in Figure 8 is quite good. The results of the deconvolution of a sub- gaussian signal using extended infomax as shown in Figures 9, 10, and 11.

\section{CONCLUSION}

In this paper we have analyzed the Infomax, Extended Infomax and Muti-user Kurtosis algorithms. The algorithms were tested based on BSS and certain relevant parameters like cross-correlation coefficient 
Figure 2. Original source signals
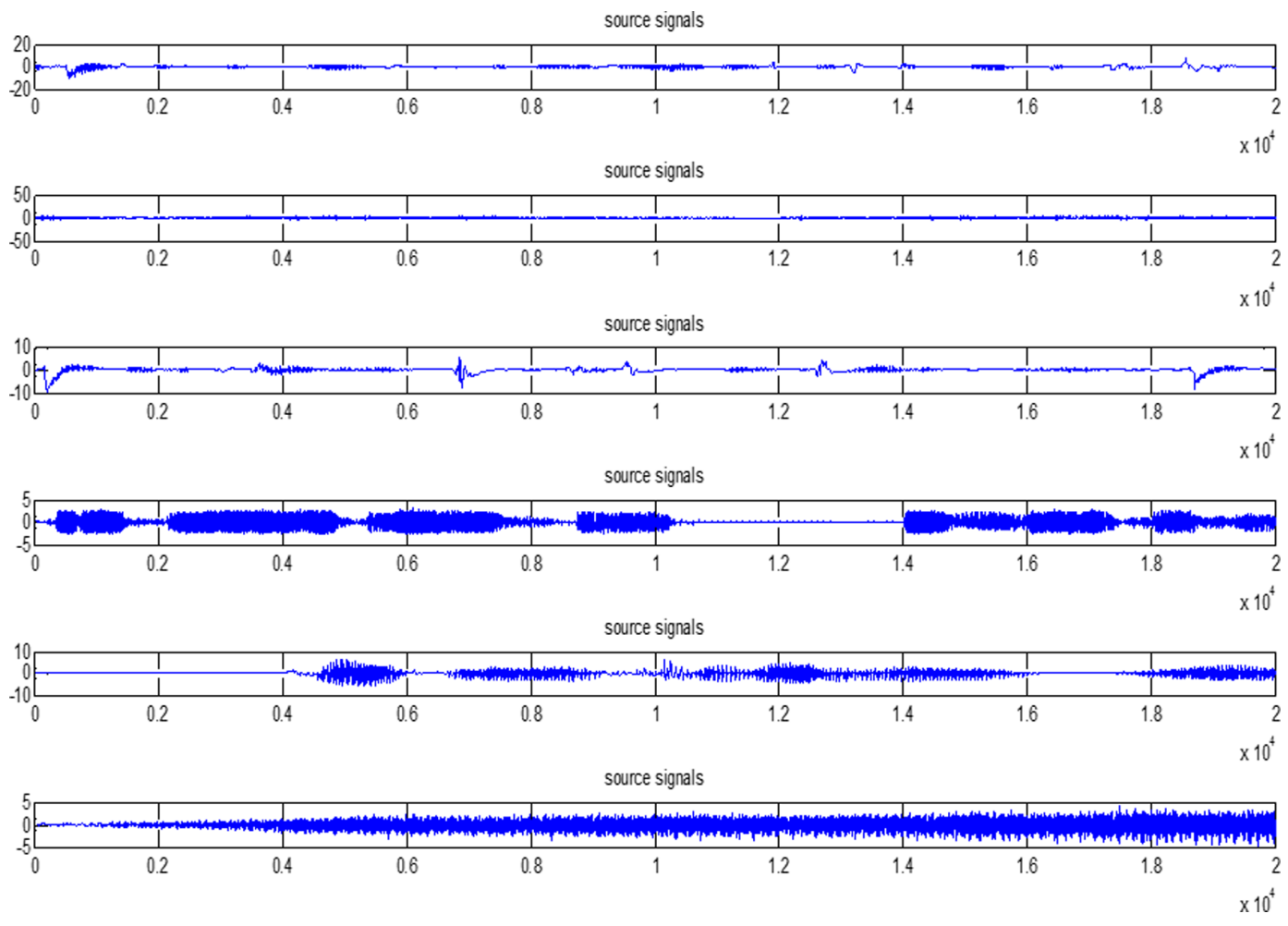

and Kurtosis were determined. Our study indicates that the Extended Infomax algorithm shows superior characteristic when compared to the others. A case study is also presented for deconvolution of different signals using Infomax and Extended Infomax. BSS techniques do not provide satisfactory performance in noisy signals and environment. As issues i.e., non-stationary sources and convolutive mixture creates problem for separation in real time.

\section{ACKNOWLEDGMENT}

The authors are grateful for the valuable input given by Prof. J. Talukdar, Silicon Institute of Technology, Bhubaneswar, Odisha. 
International Journal of Rough Sets and Data Analysis

Volume 6 • Issue 1 • January-March 2019

Figure 3. Mixture signals

mixture signals

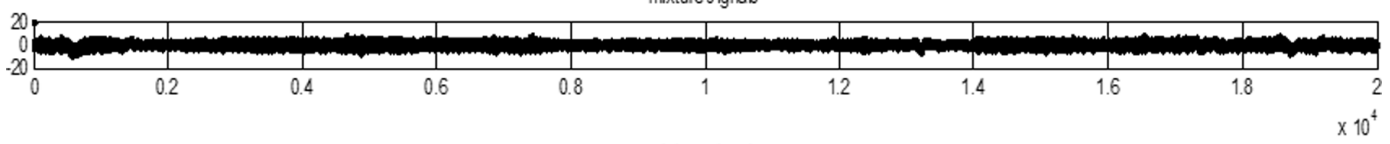

mixture signak

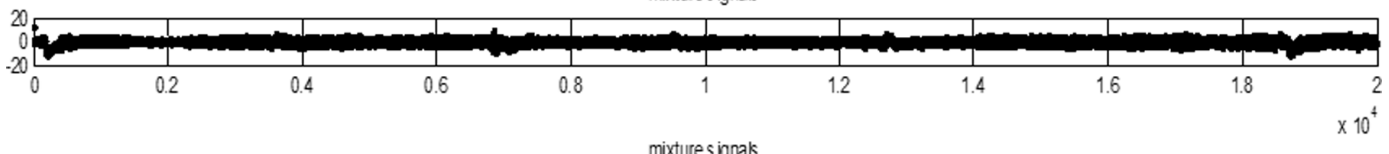

mixture signak

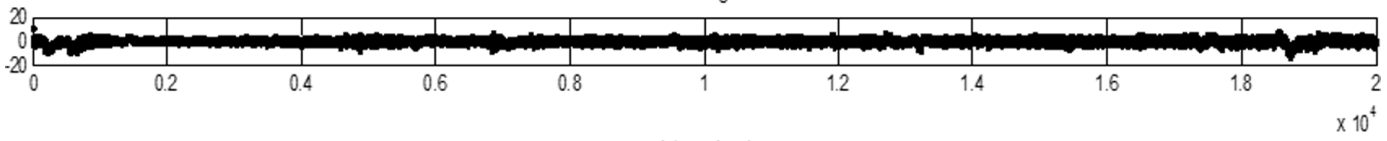

mixture signals

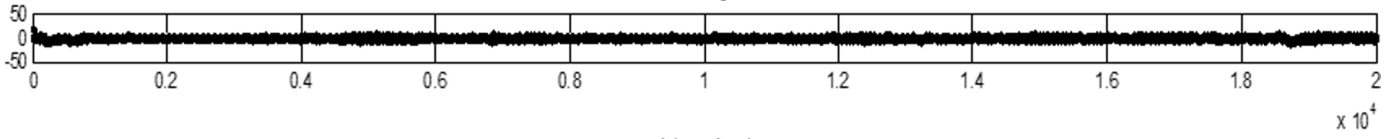

mixture signals

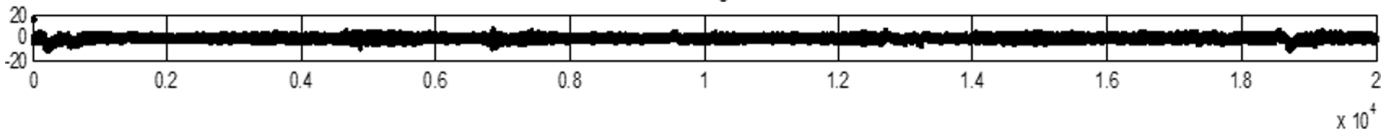

mixture signals

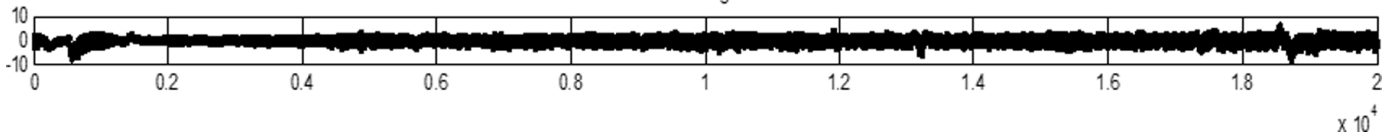

Figure 4. Extracted source signals

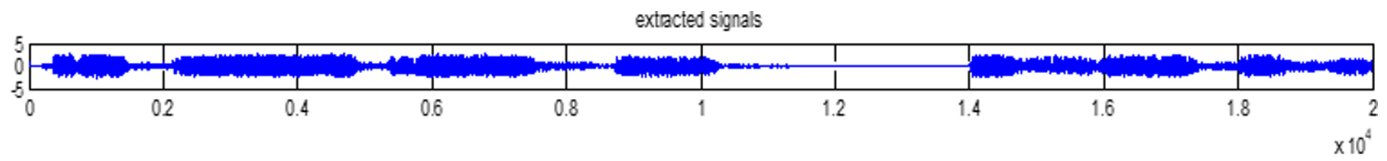

extracted signals

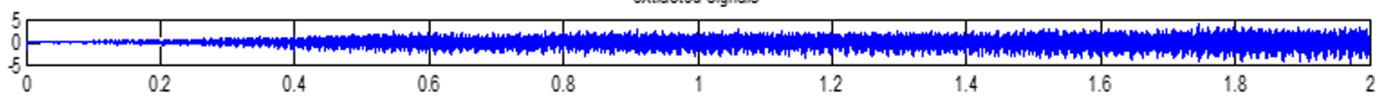

extracted signals

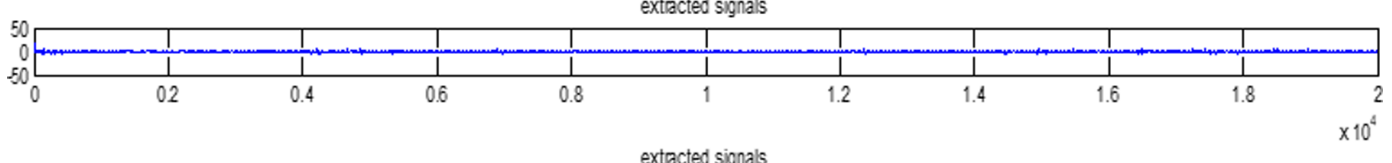

extracted signals

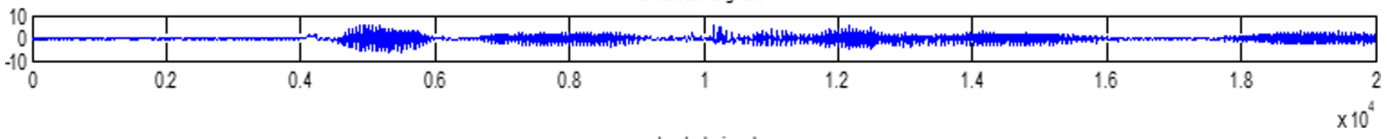

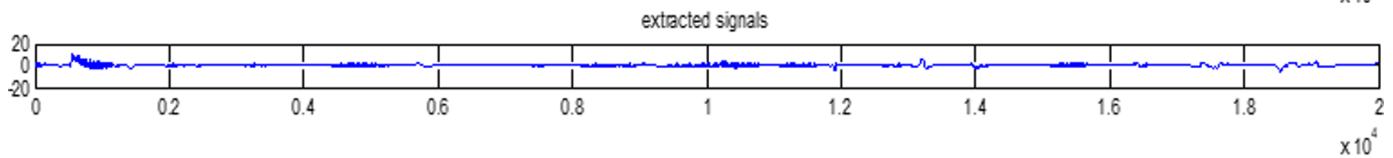

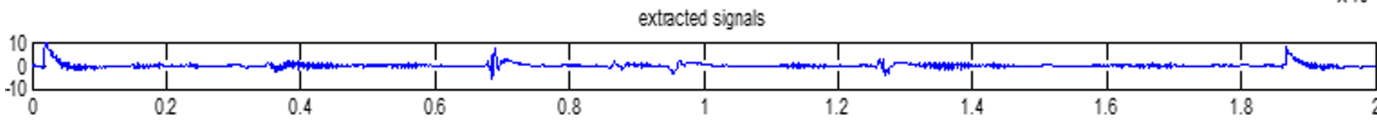


Figure 5. Blind deconvolution results of Infomax algorithm

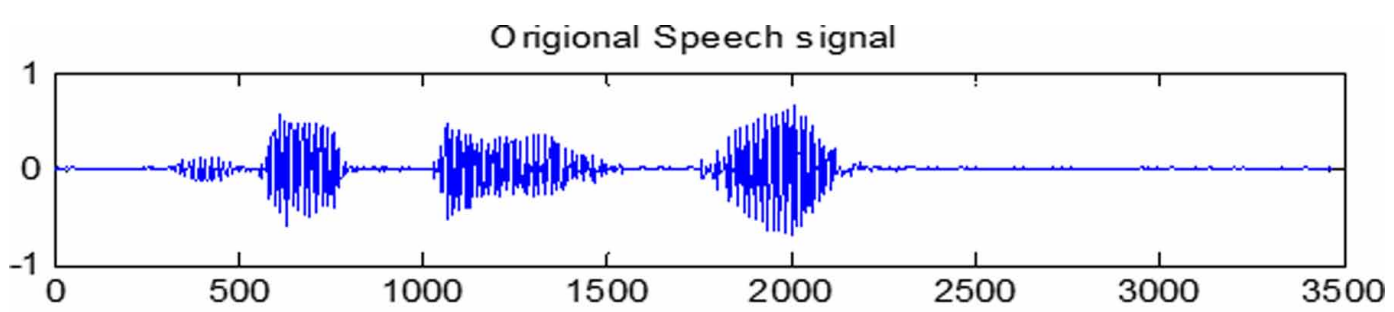

Echo corrupted signal

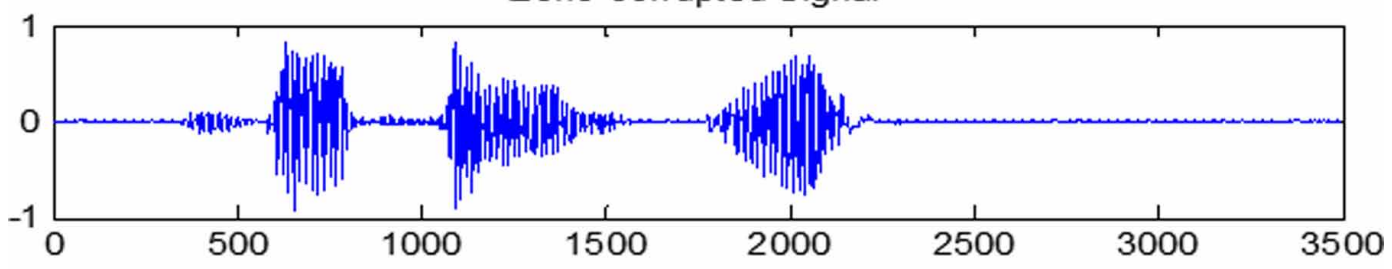

Output (Deconvolved)Signal

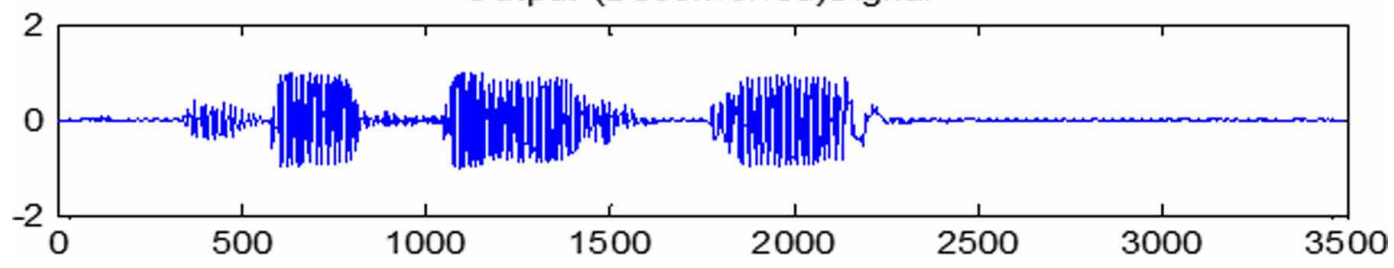

Figure 6. Blind deconvolution results of Infomax algorithm
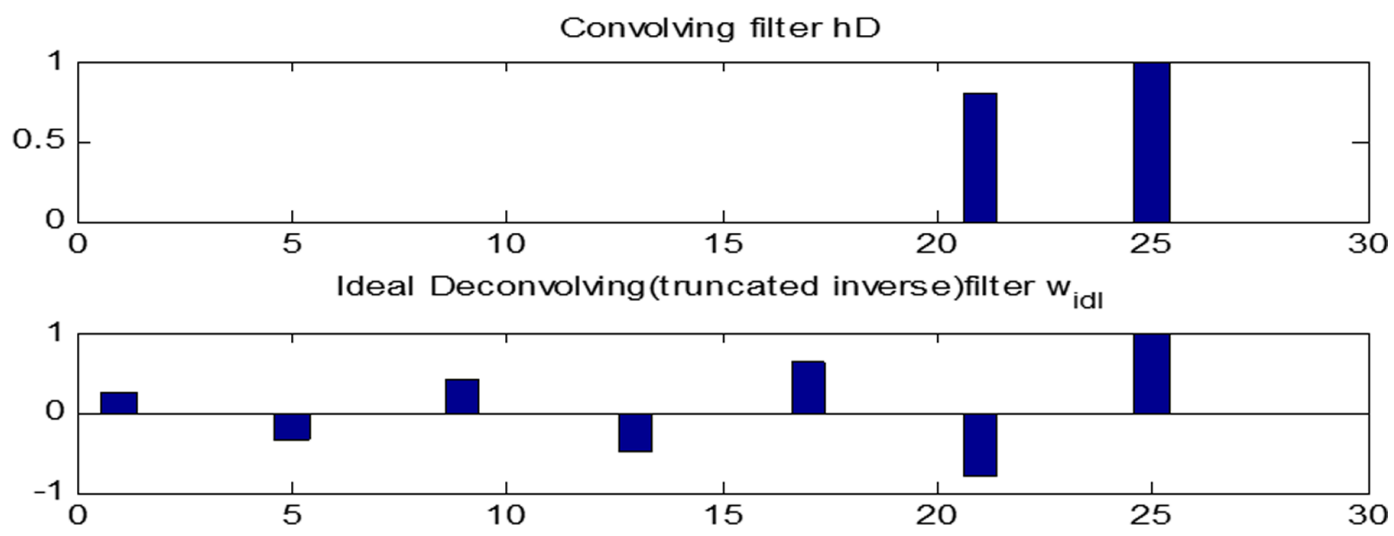

Leamt Deconvolving filter w

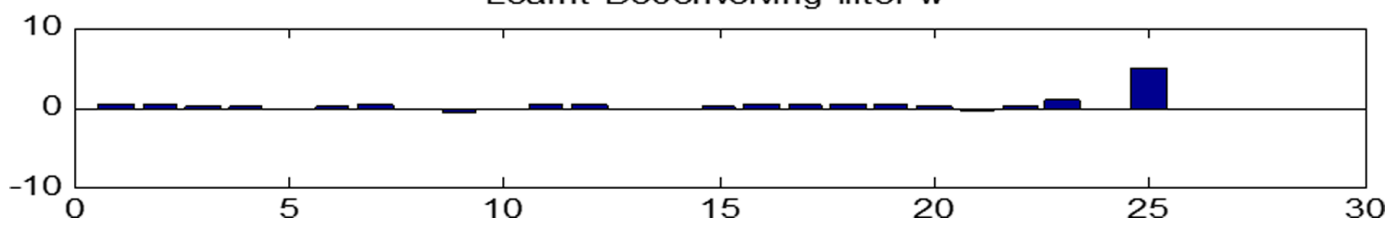


Figure 7. Blind deconvolution results of Infomax algorithm
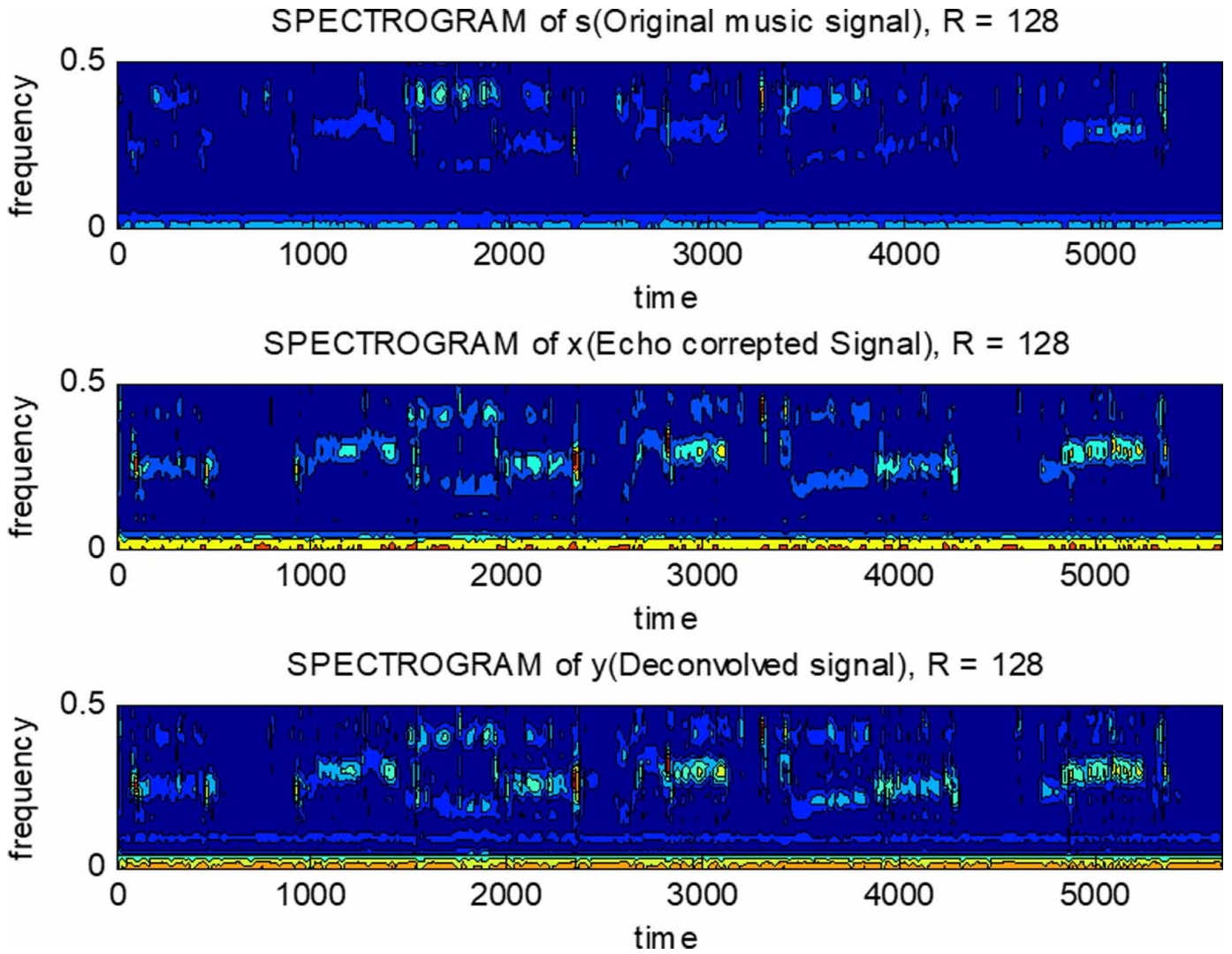
Figure 8. Blind deconvolution results of the convoluting filter for Extended Infomax algorithm

Convolving filter $\mathrm{hD}$
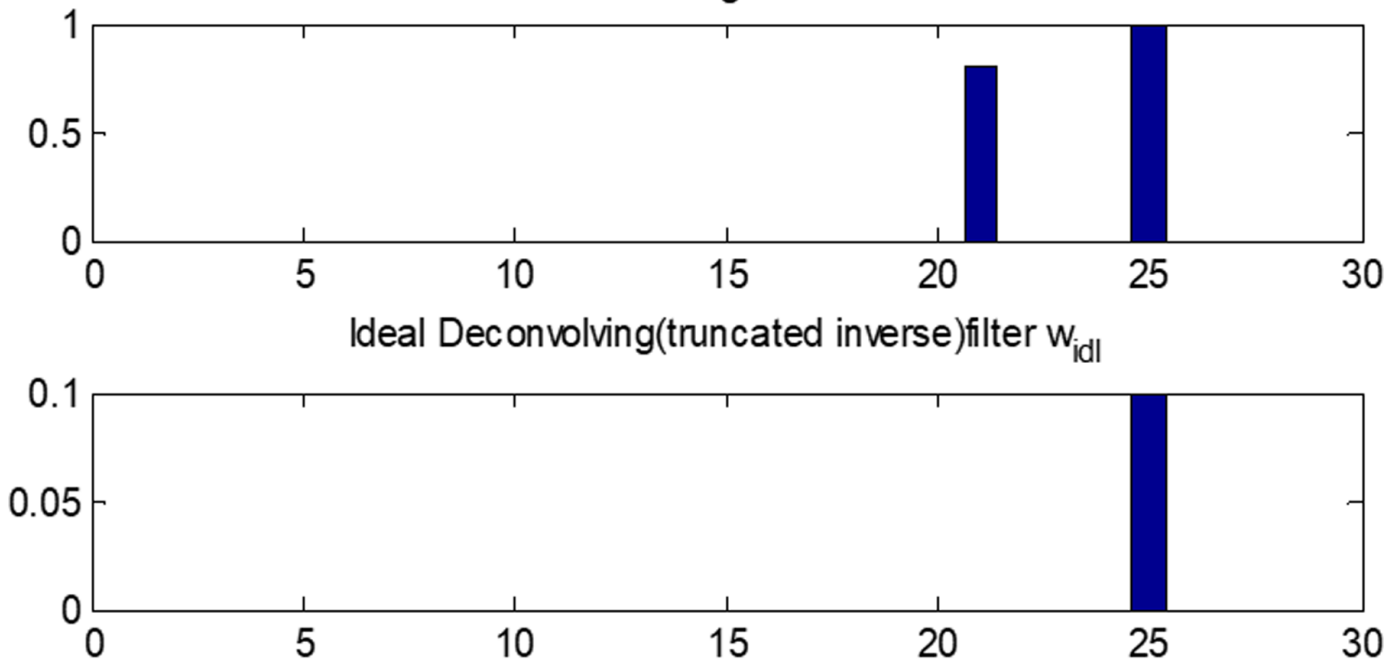

Leamt Deconvolving filter w

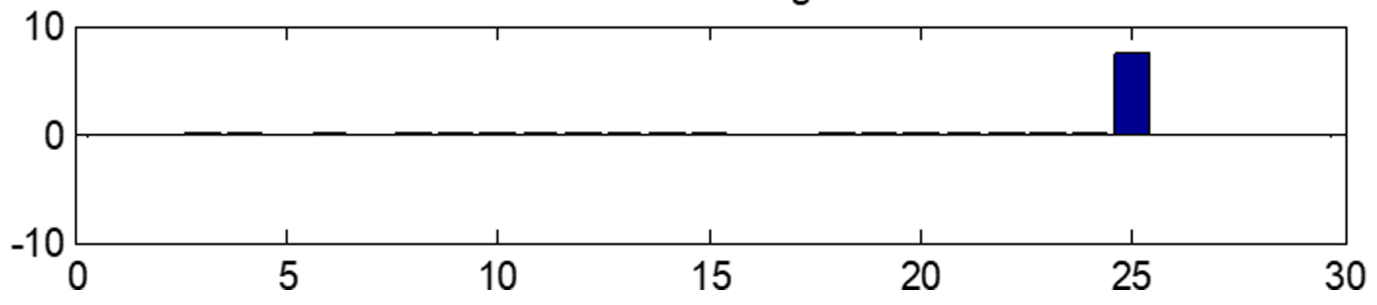


Figure 9. Results of the deconvolution of a sub- gaussian signal using extended infomax

Origional music signal
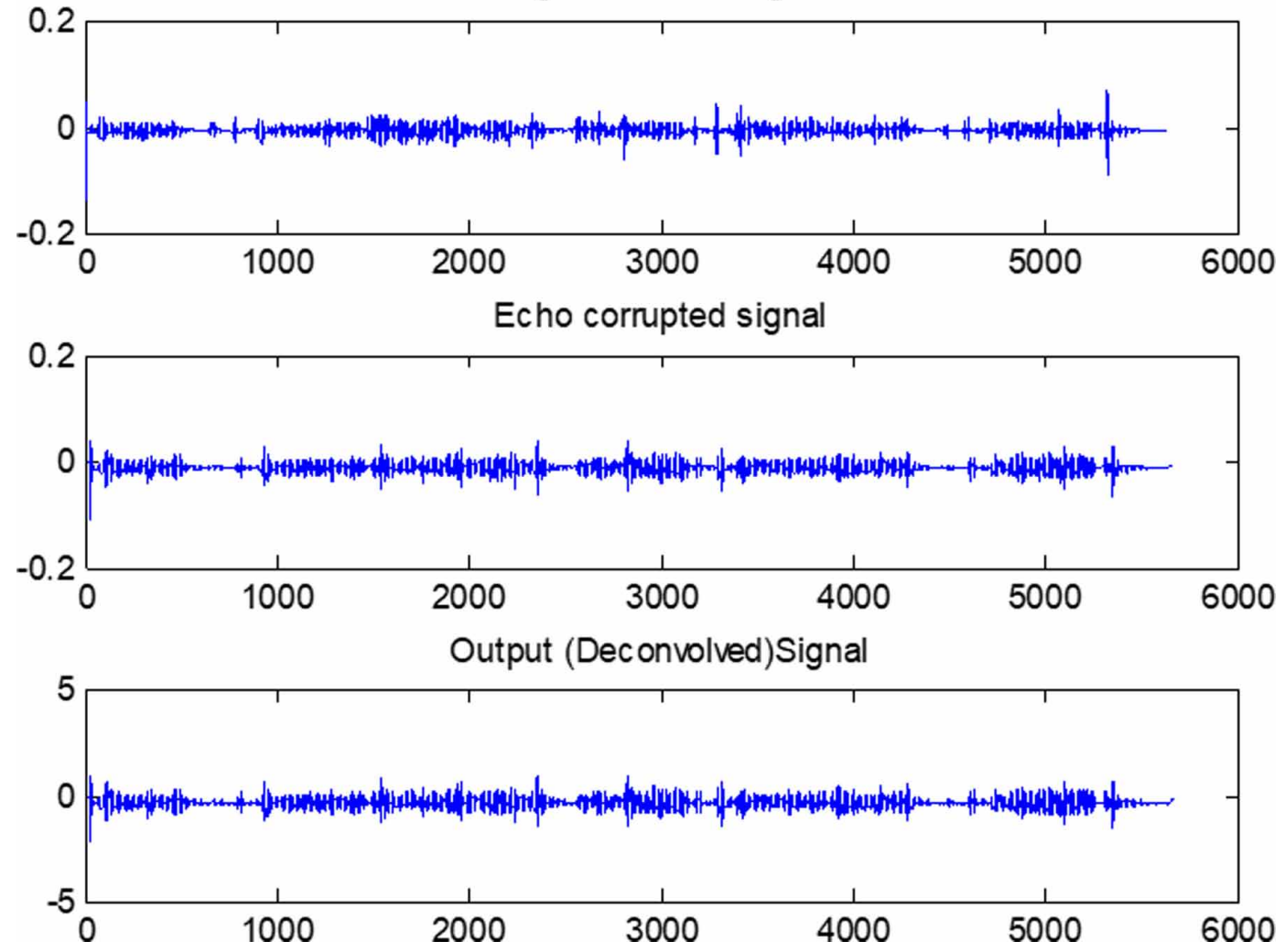
Figure 10. Results of the deconvolution of a sub- gaussian signal using extended infomax

Convolving filter $\mathrm{hD}$
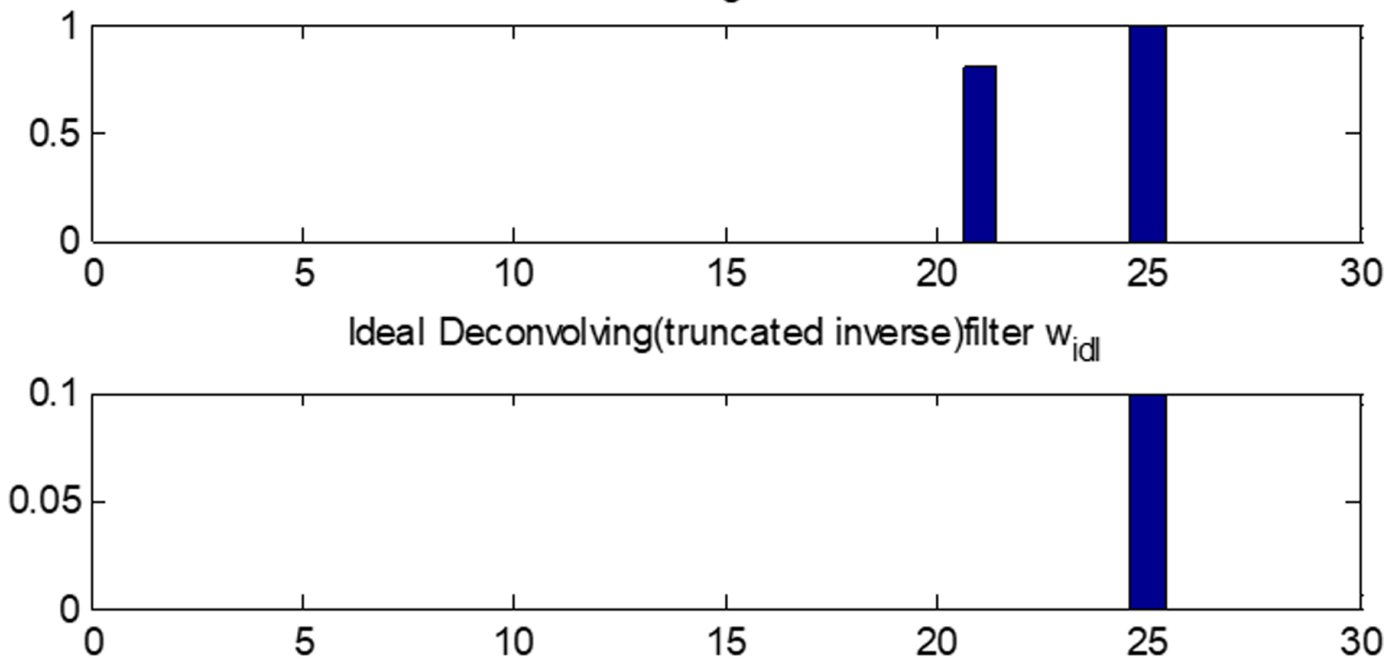

Learnt Deconvolving filter w

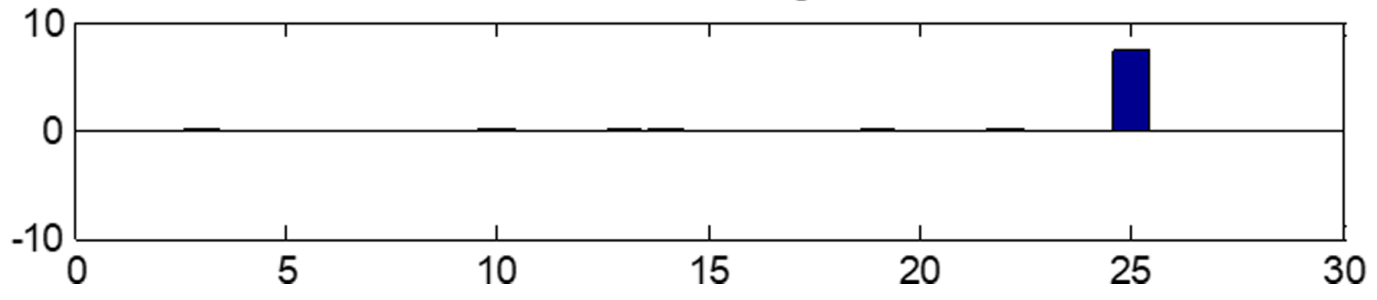


International Journal of Rough Sets and Data Analysis

Volume 6 • Issue 1 • January-March 2019

Figure 11. Results of the deconvolution of a sub- gaussian signal using extended infomax
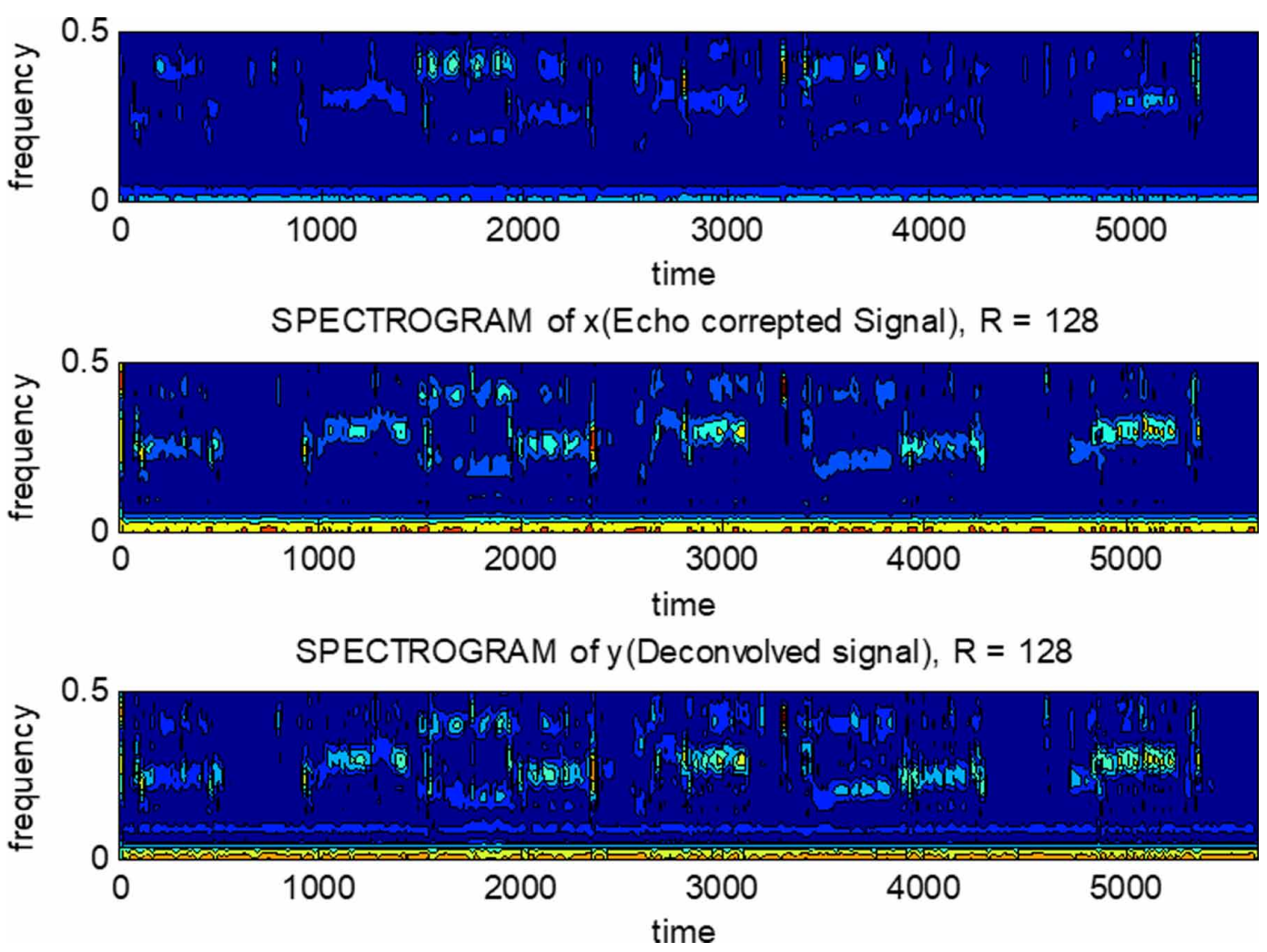


\section{REFERENCES}

Amari, S. (1998). Natural gradient works efficiently in learning. Neural Computation, 10(2), 251-27. doi:10.1162/089976698300017746

Bell, A., \& Sejnowski, T. (1995). Fast blind separation based on information theory. Neural Computation, 7 , 1129-1159. doi:10.1162/neco.1995.7.6.1129 PMID:7584893

Bell, A. J., \& Sejnowski, T. J. (1995-97, July). An Information Maximization Approach to Blind Separation and Blind Deconvolution. Neural Computation, 7(6), 1129-1159. doi:10.1162/neco.1995.7.6.1129 PMID:7584893

Comon, P. (1994). Independent Component Analysis, A new concept. Signal Processing, 36(3), 287-314. doi:10.1016/0165-1684(94)90029-9

Comon, P., \& Rota, L. (2003, March). Blind separation of independent sources from convolutive mixtures. IEICE Trans. on Fundamentals, E86-A(3), 542-549.

Das, N., Routray, A., Dash, P. K., \& India, D. (2007). ICA methods for blind source separation of instantaneous mixtures: A case study. Neural Information Process. Letters and Reviews, 11(11), 225-246.

Diamantaras, K., Vranou, G., \& Papadimitriou, T. (2013, June 1). Multi Input Single Output Nonlinear Blind separation of Binary Sources. IEEE Transactions on Signal Processing, 61(11), 2866-2873. doi:10.1109/ TSP.2013.2255046

Kadou, M., \& Arakawa, K. (2009). A Method of Blind Source Separation for Mixed Voice Separation in Noisy and Reverberating Environment. IEICE Tech. Rep., 108(461), 55.

Lee, T.-W., Girolami, M., \& Sejnowski, T. J. (1999). Independent component analysis using an Extended Infomax Algorithm for mixed sub-gaussian and Super-gaussian sources. Neural Computation, 11(2), 409-433. doi:10.1162/089976699300016719 PMID:9950738

Minhas, S. F., \& Gaydecki, P. (2014). A hybrid algorithm for blind source separation of a convolutive mixture of three speech sources. EURASIP Journal on Advances in Signal Processing, (1), 92.

Papadias, C. B. (2000, December). Globally Convergent Blind Source Separation Based on a Multiuser Kurtosis Maximization Criterion. IEEE Transactions on Signal Processing, 48(12), pp3508-pp3519. doi: $10.1109 / 78.887044$

Swain, M., Biswal, R., Panda, R., \& Kabisatpathy, P. (2017). Study of a Multiuser Kurtosis Algorithm and an Information Maximization Algorithm for Blind Source Separation. In Computational Intelligence in Data Mining (pp. 619-631). Springer. 